\title{
Optimal Topology Configuration Design of Contoured-Beam Mesh Reflectors
}

\author{
Guigeng Yang $\mathbb{D}^{1},{ }^{1}$ Aofei Tang, ${ }^{1}$ Yiqun Zhang, $^{2}$ Zhenyi Yuan, ${ }^{1}$ and Lingfei Kong ${ }^{1}$ \\ ${ }^{1}$ School of Mechanical and Precision Instrument Engineering, Xi'an University of Technology, Xi'an, Shaanxi 710048, China \\ ${ }^{2}$ School of Mechano-Electronic Engineering, Xidian University, Xi'an, Shaanxi 710071, China \\ Correspondence should be addressed to Guigeng Yang; guigengyang@xaut.edu.cn
}

Received 19 August 2021; Accepted 4 February 2022; Published 27 February 2022

Academic Editor: Giovanni Andrea Casula

Copyright (C) 2022 Guigeng Yang et al. This is an open access article distributed under the Creative Commons Attribution License, which permits unrestricted use, distribution, and reproduction in any medium, provided the original work is properly cited.

It is significant for the practical application of space-borne mesh-shaped reflectors to reduce the structural complexity under the condition of the far-field electromagnetic (EM) performance being satisfied. To address this problem, an optimal topology configuration design method is proposed for the mesh-shaped reflectors. First, a multifrequency optimization model is established to obtain the mesh-shaped reflector. The reflector nodal deviations in the $x$-, $y$-, and $z$-directions are simultaneously selected as design variables to design the shape of the mesh reflector and realize the required contoured beams within the whole frequency band. Then, based on the multifrequency optimization model, an iterative design technology is proposed to obtain the optimal topology configuration of the mesh-shaped reflector. Finally, a typical example of a mesh reflector with contoured beams covering the continental United States (CONUS) is investigated to demonstrate the feasibility of the method.

\section{Introduction}

Contoured-beam reflectors are widely used in modern satellite communication applications to improve power utilization efficiency and cover the required areas [1]. Because the array-feed approach has expensive and complex beamforming networks, single-feed-shaped reflector antennas are attractive for producing contoured beams [2-4]. On the other hand, there is a great demand for high-gain space-borne reflector antennas in future space applications. The large-aperture deployable mesh reflector antenna, which has the advantages of light mass, large aperture, and low folded volume $[5,6]$, has become one typical kind of space reflector to realize a high gain. Therefore, the contouredbeam synthesis based on mesh reflectors has been a meaningful research hotspot.

Most of the current research [2-4] on single-feed shaped reflectors focuses on solid antennas because the shaped solid reflectors can be easily manufactured and supported through structure design. However, the beam gains are limited due to the aperture size of the solid antennas. To achieve high-gain contoured beams, the research on the contoured-beam synthesis for large-aperture mesh reflectors is essential and significant.

Some related research has been conducted for the contoured-beam synthesis of mesh reflectors. A beamforming design method is presented in [7] for large-aperture mesh reflectors based on the reflecting surface expansion method. The shaped reflector is obtained by designing the coefficients of some spline functions, and the mesh-shaped reflector is obtained through the mapping relation of the aperture plane. In [8], an iterative design strategy that combines the plane-wave method and force density method is proposed for the contoured-beam synthesis of mesh reflectors. However, in these methods, the approximation processes will inevitably lead to a certain deviation between the far-field pattern of a mesh-shaped reflector and that of the ideal-shaped one. For this problem, an optimization method based on sensitivity analysis is presented in $[9,10]$, in which the reflector nodal deviations in the $z$-direction are designed to obtain a mesh-shaped reflector. Unfortunately, the obtained concave-convex mesh reflector cannot be effectively supported based on the traditional 2-layered mesh reflector antenna structures. To address this problem, a 3- 
layered cable net structure with a shaped mesh reflector is proposed in [11] to realize the force balance of the concaveconvex reflector, as shown in Figure 1. Based on the approach in [11], an improved design technique is presented in [12] to improve the deployment characteristics of the 3layered mesh reflector antenna, and a corresponding prototype is developed to verify the validity of the 3-layered cable net structure approach.

However, although the previous research studies indicate the feasibility and effectiveness of the 3-layered mesh antenna approach, there are still two problems to be solved. On the one hand, in the previous contoured-beam synthesis models, the frequency characteristics of mesh-shaped reflectors are not taken into consideration, and that only the reflector nodal deviations in the $z$-direction are selected as design variables with the nodal coordinates in $x$ - and $y$ directions being unchanged, which limits the maximum beamforming ability of mesh reflectors. On the other hand, all the existing methods focus on the shape design of mesh reflectors with given topology but have not thoroughly studied how to obtain the optimal topology of a meshshaped reflector under the condition of the far-field EM performance being satisfied.

To address the above problems, a multifrequency contoured-beam synthesis model is firstly established, in which the frequency characteristics of mesh-shaped reflectors are taken into consideration to ensure practicability in engineering. Then, based on the multifrequency optimization model, an iterative design strategy is proposed to reduce the structural complexity and obtain the optimal topology configuration of mesh-shaped reflectors under the condition of the far-field EM performance being satisfied. Finally, a typical example is investigated to illustrate the feasibility and effectiveness of the method.

\section{Methodology}

2.1. Contoured-Beam Synthesis Technology considering Frequency Characteristics. In this section, the mesh-shaped reflector is firstly designed to generate the required contoured beams with the frequency characteristics being taken into consideration.

If the topology configuration and nodal coordinates of a mesh reflector are given, the far-field pattern can be figured out by the Physical Optics method [11].

$$
\vec{E}(\theta, \phi)=-j k \eta \frac{e^{-j k r}}{4 \pi r}(\widehat{\widehat{I}}-\widehat{r} \widehat{r}) \cdot \iint_{\Sigma} \vec{J}\left(\vec{r}^{\prime}\right) e^{j k r \cdot \overrightarrow{\mathbf{r}}^{\prime}} \mathrm{d} \Sigma^{\prime},
$$

where $\vec{E}(\theta, \phi)$ is the electric field intensity at the far-field observation direction $(\theta, \phi), j=\sqrt{-1}, k=2 \pi / \lambda$ ( $\lambda$ is the wavelength), $\eta=120 \pi, \vec{J}\left(\vec{r}^{\prime}\right)=2 \widehat{n} \times \vec{H}^{i}$ is the induced current, $\widehat{n}$ is the outward unit normal vector, $\vec{H}^{i}$ is the incident magnetic field, $\Sigma$ is the curved reflector surface, $\vec{r}^{\prime}$ is the integration point vector of the reflector, and $\hat{r}$ is the unit vector of the observation direction, as shown in Figure 2.

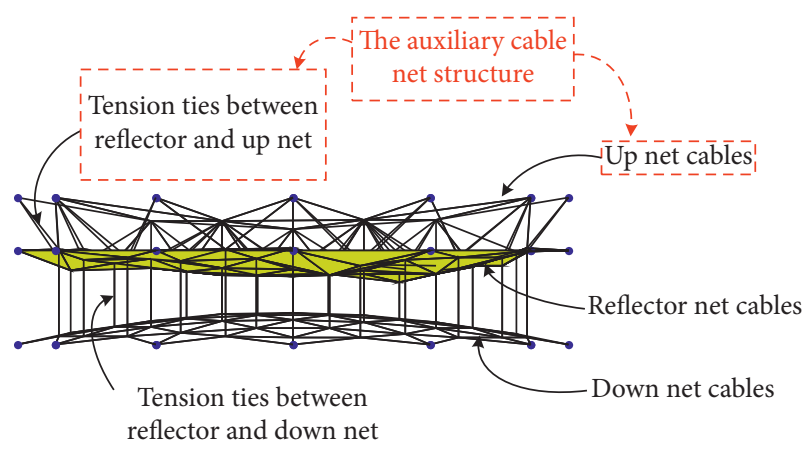

Figure 1: A 3-layered cable net structure with a mesh-shaped reflector. An auxiliary layer of cable net structure is added to the traditional 2-layered AstroMesh structure to realize the bidirectional force on the reflector.

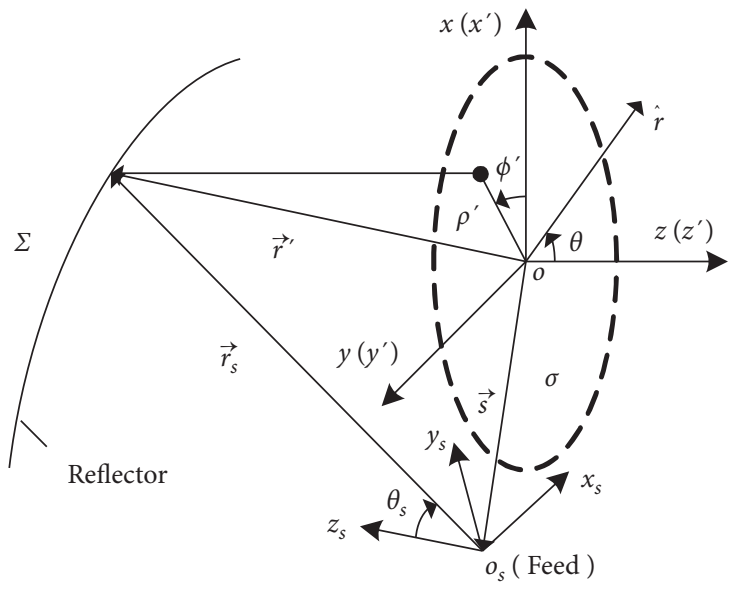

Figure 2: A reflector antenna illuminated by an arbitrarily located feed.

In this study, an idealized cosine-Q feed with a fixedphase center is adopted to radiate a linear-polarized wave, whose electric field vector can be expressed as follows: [13]

$$
\begin{aligned}
\vec{E}_{s}\left(\vec{r}_{s}\right)=A_{0}\left\{\begin{array}{l}
\hat{\theta}_{s} C_{E}\left(\theta_{s}\right) \cos \phi_{s}-\widehat{\phi}_{s} C_{H}\left(\theta_{s}\right) \sin \phi_{s} \\
\hat{\theta}_{s} C_{E}\left(\theta_{s}\right) \sin \phi_{s}+\widehat{\phi}_{s} C_{H}\left(\theta_{s}\right) \cos \phi_{s}
\end{array}\right\} \\
\cdot \frac{e^{-j k r_{s}}}{r_{s}}\left\{\begin{array}{l}
\hat{x} \text { polarized } \\
\hat{y} \text { polarized }
\end{array}\right.
\end{aligned}
$$

where

$$
\left\{\begin{array}{l}
C_{\mathrm{E}}\left(\theta_{\mathrm{s}}\right)=\left(\cos \theta_{\mathrm{s}}\right)^{q \mathrm{E}}, \quad \mathrm{E}-\text { plane pattern, } \\
C_{\mathrm{H}}\left(\theta_{\mathrm{s}}\right)=\left(\cos \theta_{\mathrm{s}}\right)^{q \mathrm{E}}, \quad \mathrm{H}-\text { plane pattern, } \\
A_{0}=\frac{1}{(4 \pi)},
\end{array}\right.
$$


For mesh-shaped reflector antennas, the far-field directivity is generally used as its design index. The far-field directivity can be expressed as follows [12]:

$$
\begin{aligned}
D(\theta, \phi) & =\frac{|\vec{E}(\theta, \phi)|^{2}}{P_{r_{s}} / 4 \pi r_{s}^{2}}, \\
P_{r_{s}} & =\int_{0}^{\pi / 2} \int_{0}^{2 \pi}\left|\vec{E}_{s}\left(\theta_{s}, \phi_{s}\right)\right|^{2} r_{s}^{2} \sin \theta_{s} \mathrm{~d} \theta_{s} \mathrm{~d} \phi_{s},
\end{aligned}
$$

where $P_{r_{s}}$ is the total radiation power of the feed, $\vec{E}_{s}\left(\theta_{s}, \phi_{s}\right)$ is the radiation electric field intensity at the observation direction $\left(\theta_{s}, \phi_{s}\right)$ in the feed coordinate system, and $r_{s}$ is the distance between a radiation point and the feed.

For a mesh reflector with a given topology configuration, the reflector nodal coordinates can be optimized to obtain a shaped mesh reflector according to [11]. In this work, the nodal deviations between the designed mesh reflector and the initial ideal one in $x$-, $y$-, and $z$-directions are simultaneously selected as design variables, which theoretically expands the design space compared with the method in [11] and is beneficial to reduce the structural complexity of the obtained mesh-shaped reflectors. Consequently, a multifrequency optimization model is established.

PI:

$$
\text { Find } \begin{aligned}
\Delta \mathbf{r} & =[\Delta \mathbf{x}, \Delta \mathbf{y}, \Delta \mathbf{z}]^{T} \\
& =\left[\Delta x_{1}, \ldots, \Delta x_{n}, \Delta y_{1}, \ldots, \Delta y_{n}, \Delta z_{1}, \ldots, \Delta z_{n}\right]^{T},
\end{aligned}
$$

$$
\operatorname{Min} F_{f}=\sum_{k=1}^{N_{k}} w_{k} f_{k}
$$

S.t. $\quad \Delta \underline{x} \leq \Delta x_{j} \leq \Delta \bar{x},(j=1 \sim n)$,

$$
\begin{aligned}
& \Delta \underline{y} \leq \Delta y_{j} \leq \Delta \bar{y},(j=1 \sim n), \\
& \Delta \underline{z} \leq \Delta z_{j} \leq \Delta \bar{z},(j=1 \sim n), \\
& D_{k i} \geq \underline{D},\left(i=1 \sim N_{\mathrm{far}}, k=1 \sim N_{k}\right) .
\end{aligned}
$$

In (6), $F_{f}$ is the comprehensive objective of the far-field electromagnetic performance with the frequency characteristics being taken into consideration. In this study, to consider the frequency characteristics of mesh-shaped reflectors, the frequency bandwidth of the antenna is discretized into $N_{k}$ frequency points. $f_{k}\left(k=1 \sim N_{k}\right)$ is the design objective at the $k$ th operating frequency point, and $w_{k}$ is the corresponding weight coefficient. $f_{k}$ can be figured out as follows:

$$
f_{k}=\left[\frac{1}{N_{\mathrm{far}}} \sum_{i=1}^{N_{\mathrm{far}}} \omega_{i}\left(D_{k i}-D^{\mathrm{obj}}\right)^{2}\right]^{1 / 2},
$$

where $N_{\text {far }}$ is the number of far-field observation directions, $D^{\text {obj }}$ is the desired object directivity, $D_{k i}$ is the directivity of the $i$ th far-field observation direction at the $k$ th operating frequency point, and $\omega_{i}$ is the corresponding selective coefficients of directivity errors and can be determined as follows:

$$
\omega_{i}= \begin{cases}1, & D_{k i}<D^{\mathrm{obj}} \\ 0, & D_{k i} \geq D^{\mathrm{obj}}\end{cases}
$$

In Equations (7)-(9), $n$ is the number of reflector free nodes and $\Delta x_{j}=x_{j}-x_{j}^{\text {initial },} \Delta y_{j}=y_{j}-y_{j}^{\text {initial }}$, and $\Delta z_{j}=z_{j}-z_{j}^{\text {initial }}$, where $x_{j}^{\text {initial }}, y_{j}^{\text {initial }}$, and $z_{j}^{\text {initial }}$ are the $x-, y$ , and $z$-coordinates of node $j$ on the initial ideal parabolic surface and $\Delta \underline{x}, \Delta \bar{x}, \Delta \underline{y}, \Delta \bar{y}, \Delta \underline{z}$, and $\Delta \bar{z}$ are the lower and upper limit values of the design variables. In (10), $\underline{D}$ is the lower limit value of the far-field directivity.

In this study, the optimization model PI can be solved through the particle swarm optimization algorithm [14].

\subsection{Topology Configuration Design of Mesh-Shaped Reflectors.} Based on the contoured-beam synthesis technology in Section 2.1, an iterative design strategy is proposed to obtain the optimal topology of mesh-shaped reflectors and the iteration design flow chart is presented in Figure 3. The detailed design procedure is described as follows.

Step 1. Determine the coverage area of the contoured beam and the desired object directivity $D^{\mathrm{obj}}$.

Step 2. Determine the basic electromagnetic and geometric parameters of the reflector: the operating frequency band $\left[f_{l}, f_{u}\right]$, central frequency $f_{0}$ of the frequency band, aperture diameter $D$, focal length $F$, and offset distance $H$.

Step 3. Generate the initial geometric configuration of an offset mesh reflector. In the example of this work, the initial geometric configuration of the mesh reflector is generated based on the quasi-geodesic mesh, which has a 6 by 6 "diamatic" pattern [15] with the main cable's segment number $N^{\text {ref }}=5$, as shown in Figure 4 .

Step 4. Establish and solve the optimization model PI to obtain a mesh-shaped reflector.

Step 5. Judge whether the far-field electromagnetic performance of the obtained mesh-shaped reflector meets the design requirements, i.e., $F_{f}^{\text {best }}=0$. If yes, reduce the number of the reflector free nodes, update the initial geometric configuration of the mesh reflector, and return to Step 4. If no, then the obtained topology configuration of the meshshaped reflector in the penultimate iteration is the optimal topology configuration.

It should be pointed out that the node reduction and mesh updating principles in Step 5 are as follows: (1) For the mesh reflector in Figure 4, only the nodes in the second and third rings can be reduced, while the nodes in the center and those on the first, outermost, and secondary outer rings remain unreduced to ensure the symmetry and mechanical 


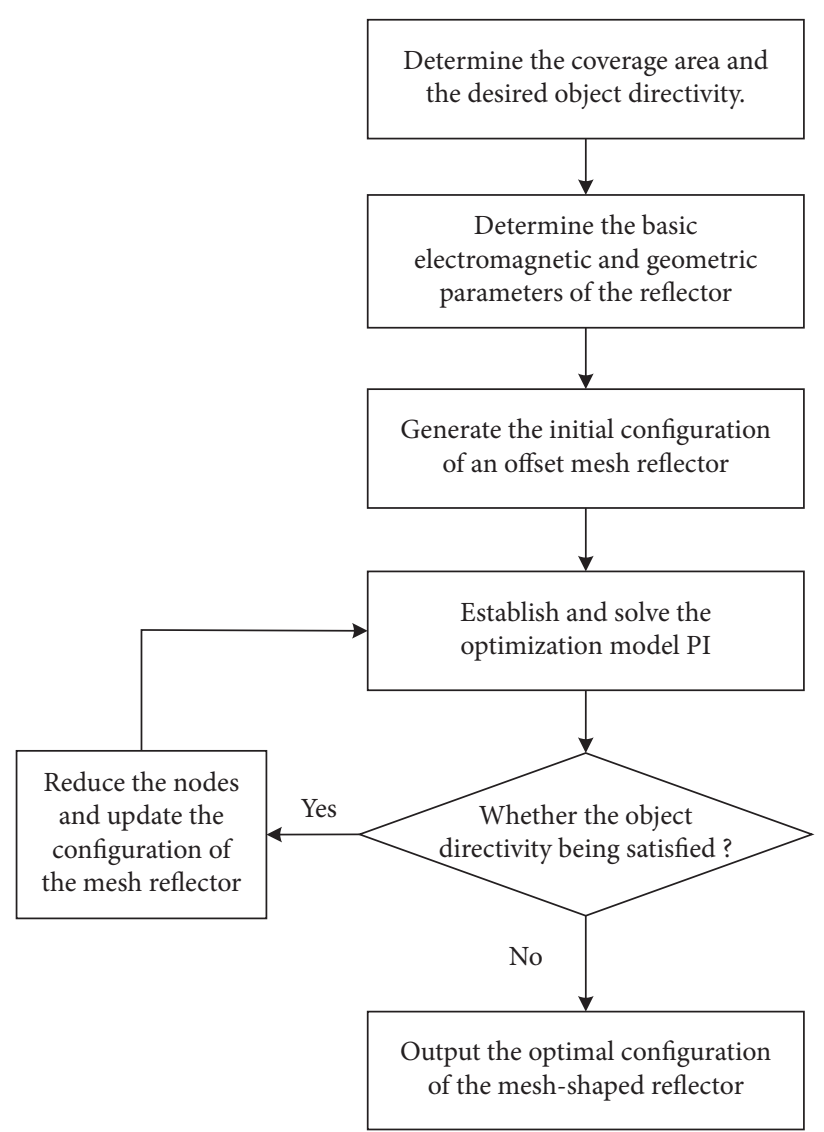

Figure 3: Iteration design flow chart.

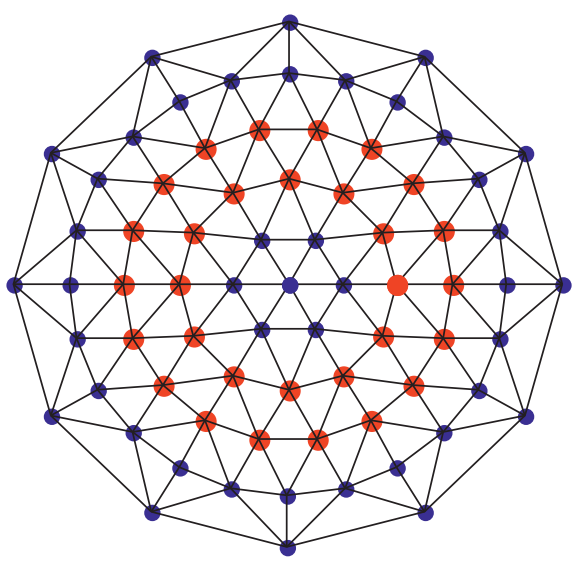

Nodes that cannot be reduced

Nodes that can be reduced

(a)

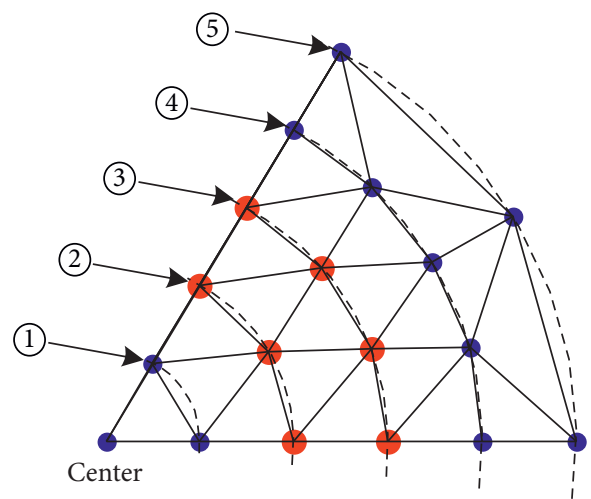

- Nodes that cannot be reduced

Nodes that can be reduced

FIGURE 4: Initial geometric configuration of a mesh reflector. (a) Classifications of the reflector nodes. (b) One-sixth model of the reflector. Note. (1) is a node on the first ring, (2) is a node on the second ring, (3) is a node on the third ring, and (4) and (5) are the nodes on the secondary outer and outermost rings, respectively.

characteristics of the mesh antenna structure. (2) Once a node on any ring is deleted, the remaining nodes should be evenly distributed on the circumference, and then, the reflector topology configuration is updated through the Delaunay triangulation. (3) In the process of node reduction, if the total number of remaining nodes in the second and third rings is fewer than half of the initial total number, the two rings should be merged into one ring.

For the example of this work, if the initial mesh reflector in Figure 4 is reduced by 19 nodes, the updated reflector 
topology configuration can be obtained, as shown in Figure 5.

\section{Numerical Results and Discussion}

The typical example in [11] is investigated to compare the results of different methods and demonstrate the effectiveness of the proposed approach. The geometric specifications of the mesh reflector are as follows: aperture diameter $D=2.5 \mathrm{~m}$, focal length $F=2.5 \mathrm{~m}$, and distance of the aperture center from the paraboloid axis $H=1.55 \mathrm{~m}$. The parameters of the cosine-Q feed are adopted as $q_{\mathrm{E}}=q_{\mathrm{H}}=11.25$. The operating frequency band is $[2.55,3.45]$ $\mathrm{GHz}$, and the central frequency point of the band is $3 \mathrm{GHz}$. The contoured beams within the whole frequency band are required to cover the CONUS map, and the desired object directivity $D^{\text {obj }}$ is required to be $28 \mathrm{dBi}$. The configuration of the initial mesh reflector is shown in Figure 4.

3.1. Obtained Mesh-Shaped Reflector. The optimization model PI and iterative design strategy in Section 2 are employed to obtain the optimal topology configuration of the mesh-shaped reflector. In this example, the lower edge, the upper edge, and the central frequencies of the frequency band are chosen to implement the multifrequency optimization, and the corresponding weight coefficients are shown in Table 1, which is the most satisfactory case with our many trials. In the contoured-beam optimization model, $\Delta \underline{x}, \Delta y$, and $\Delta \underline{z}$ are set to be $-0.8 \lambda$ and $\Delta \bar{x}, \Delta \bar{y}$, and $\Delta \bar{z}$ are set to be $0.8 \lambda$.

The far-field patterns of the obtained optimal meshshaped reflector at the lower edge, the upper edge, and the central operating frequencies are shown in Figure 6, where the black solid dots represent the observation directions in the far field. The reflector can generate the desired contoured beams covering the CONUS with the object directivity 28 $\mathrm{dBi}$ being satisfied at all three operating frequencies. In addition, in order to further show the frequency characteristics within the whole frequency band, the whole bandwidth is divided into 11 frequency points and the farfield patterns at all the 11 frequency points are calculated, as shown in Table 2. We can obtain that the mesh-shaped reflector can generate the desired contoured beams within the whole frequency band through multifrequency optimization.

Figure 7 shows the configuration of the obtained optimal mesh-shaped reflector. Figure $8(a)$ and $8(b)$ show the nodal deviations between the designed and the initial ideal parabolic surface in the oxy plane and $z$-direction, respectively. We can readily observe that the mesh distribution of the reflector changes to a certain extent in the projection plane and the obtained mesh-shaped reflector is still a concaveconvex surface.

By comparing the obtained mesh-shaped reflector with the initial mesh reflector in Figure 4, we can obtain that the structural complexity of the mesh reflector is greatly reduced. The detailed data about the node numbers, cable element numbers, and triangle element numbers of the

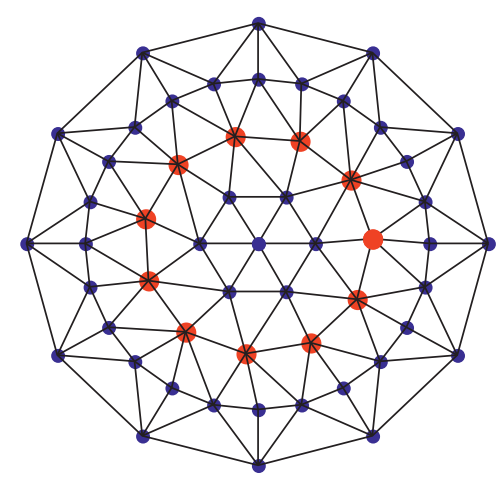

FIgURE 5: A configuration of the mesh reflector after node reduction.

TABLE 1: The weight coefficients of different operating frequency points.

\begin{tabular}{lc}
\hline Operating frequency $(\mathrm{GHz})$ & Weight coefficients \\
\hline 2.55 & $1 / 2$ \\
3.00 & $1 / 6$ \\
3.45 & $1 / 3$ \\
\hline
\end{tabular}

initial mesh reflector in Figure 4, the mesh-shaped reflector in [11], and the obtained mesh-shaped reflector in this work are listed in Table 3. Compared with the mesh-shaped reflector in [11], the numbers of nodes, cable elements, and triangle elements of the obtained one are reduced by $19.4 \%$, $22.4 \%$, and $21.7 \%$, respectively. Therefore, for the obtained mesh-shaped reflector, not only the electromagnetic performance requirements are satisfied but also the structural complexity of the mesh reflector is greatly improved.

3.2. Obtained 3-Layered Mesh-Shaped Antenna. To further illustrate the validity and realizability of the obtained meshshaped reflector, a 3-layered cable net structure based on the design approach in [11] is designed to support the obtained mesh-shaped reflector, as shown in Figure 9. Here, it should be pointed out that the aperture of the up cable net is equal to that of the reflector to reduce the design difficulty of the support rim truss in our future work. The pretension design of the obtained 3-layered cable net is implemented, and the detailed pretension results are listed in Table 4 . We can obtain that the tension range of the whole 3-layered cable net structure is from $5 \mathrm{~N}$ to $74.03 \mathrm{~N}$ and the maximum tension ratio of the cable nets is 9.25 . The tension distribution and tension level are similar to those in $[11,12]$. Therefore, the obtained 3-layered cable net structure is realizable in practical engineering.

In addition, the detailed results about the structural complexity comparison of the whole 3-layered mesh-shaped antennas in [11] and this work are listed in Table 5. According to Tables 3 and 5, with the reduction in the structural complexity of the reflector, the numbers of nodes and cable elements of the obtained whole 3-layered meshshaped antenna are also reduced by $17.0 \%$ and $20.9 \%$ compared with the results in [11]. 


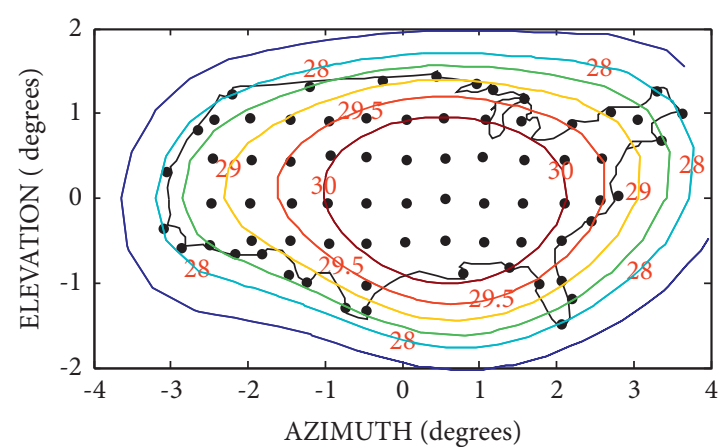

(a)

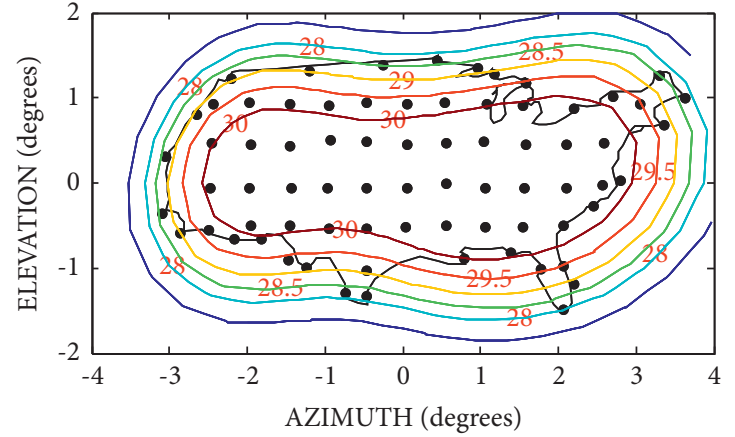

(b)

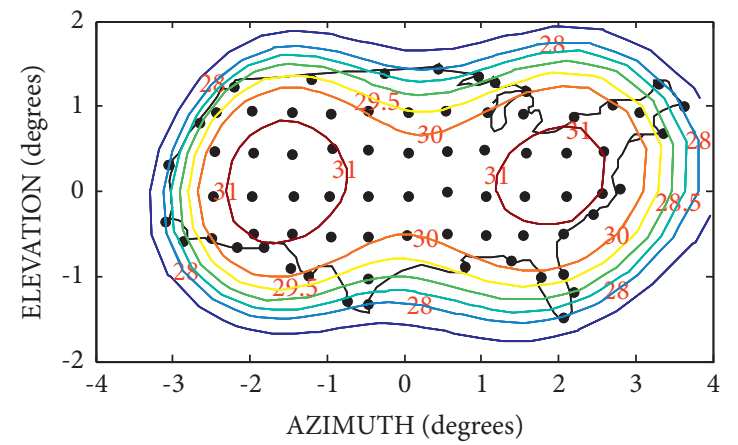

(c)

Figure 6: Far-field patterns of the obtained mesh-shaped reflector. (a) Operating frequency: $2.55 \mathrm{GHz}$. (b) Operating frequency: $3.00 \mathrm{GHz}$. (c) Operating frequency: $3.45 \mathrm{GHz}$.

TABLE 2: Far-field pattern results within the whole frequency band.

\begin{tabular}{lr}
\hline Operating frequency $(\mathrm{GHz})$ & $D_{i}(i=1-73)(\mathrm{dBi})$ \\
\hline 2.55 & $28.003-30.908$ \\
2.64 & $28.024-30.906$ \\
2.73 & $28.039-30.889$ \\
2.82 & $28.055-30.860$ \\
2.91 & $28.069-30.851$ \\
3.00 & $28.081-30.853$ \\
3.09 & $28.088-30.919$ \\
3.18 & $28.064-30.997$ \\
3.27 & $28.029-31.199$ \\
3.36 & $28.012-31.414$ \\
3.45 & $28.002-31.621$ \\
\hline
\end{tabular}

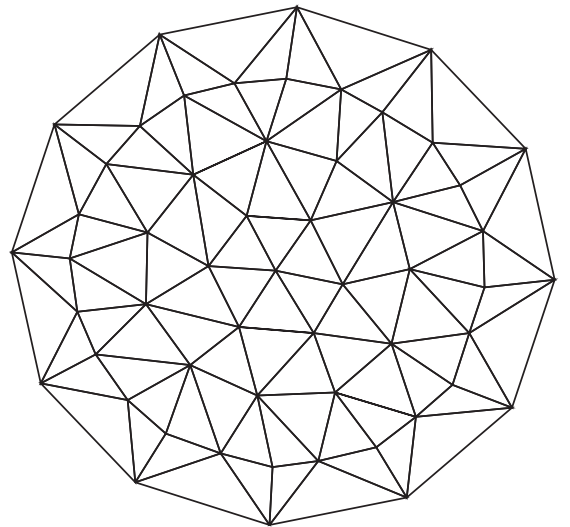

(a)

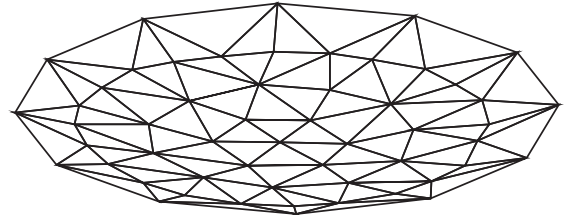

(b) (c)

Figure 7: The obtained mesh-shaped reflector. (a) Top view. (b) Isometric view. (c) Side view. 


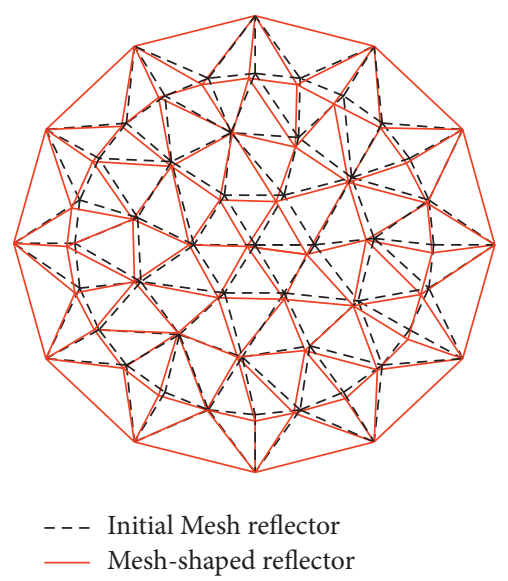

(a)

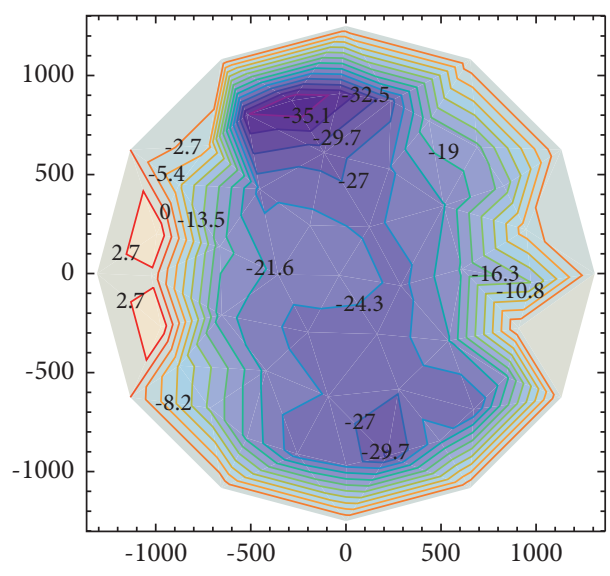

(b)

FIGURE 8: Surface nodal deviations of the mesh-shaped reflector. (a) In the oxy plane. (b) In the $z$-direction.

TABle 3: Mesh complexity comparison of the mesh reflectors before and after topology design.

\begin{tabular}{lccc}
\hline \multirow{2}{*}{ Items } & \multirow{2}{*}{ Initial mesh reflector in Figure 4} & \multicolumn{2}{c}{ Mesh-shaped reflectors } \\
& & Premethod in [11] & 67 \\
Number of nodes & 73 & 174 & 54 \\
Number of cables & 192 & 120 & 135 \\
Number of triangle elements & 132 & 94 \\
\hline
\end{tabular}

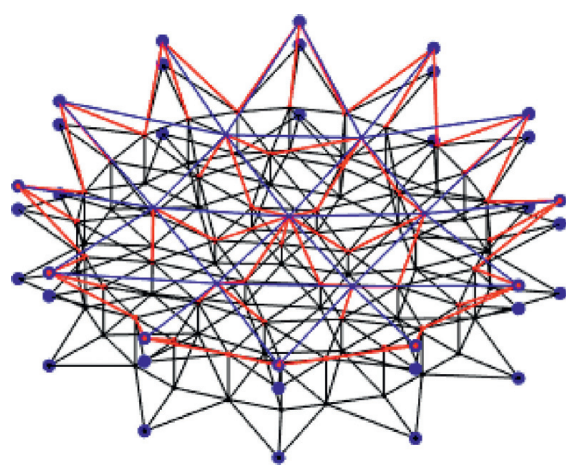

(a)

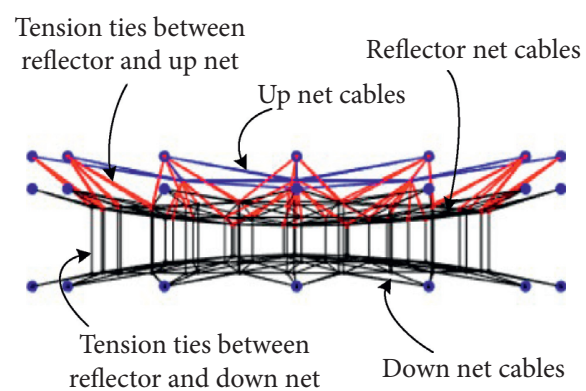

(b)

Figure 9: 3-layered cable net structure with the obtained mesh-shaped reflector. (a) Isometric view. (b) Side view.

TABle 4: Pretension distribution of the obtained 3-layered mesh antenna.

\begin{tabular}{lccc}
\hline Items & Maximum $(\mathrm{N})$ & Minimum $(\mathrm{N})$ & Maximum tension ratio \\
\hline Reflector net cables & 21.89 & 5.00 & 4.38 \\
Up net cables & 67.52 & 7.30 & 9.25 \\
Down net cables & 74.03 & 8.99 & 8.23 \\
Tension ties between the reflector and up net & 10.00 & 5.00 & 2.00 \\
Tension ties between the reflector and down net & 9.89 & 5.00 & 1.98 \\
\hline
\end{tabular}

TABLE 5: Structural complexity comparison of the 3-layered mesh-shaped antennas before and after topology design.

\begin{tabular}{lcc}
\hline Items & 3-layered mesh-shaped antennas & This work \\
\hline Number of nodes & Premethod in [11] & 123 \\
Number of cables & 512 & 405 \\
\hline
\end{tabular}




\section{Conclusions}

In this work, an optimal topology configuration design method is proposed to reduce the structural complexity of mesh-shaped reflectors. On the one hand, a multifrequency optimization model is established to obtain the mesh-shaped reflector with frequency characteristics being taken into consideration. On the other hand, an iterative design method is proposed to obtain the optimal topology configuration of the mesh-shaped reflector. Numerical simulations illustrated the effectiveness of the method. From the results and discussion, conclusions can be drawn as follows:

(1) The obtained mesh-shaped reflector can produce satisfactory contoured beams for the required coverage region within the whole frequency band through multifrequency optimization.

(2) The structural complexity of the mesh-shaped antenna is greatly reduced under the condition of the far-field electromagnetic performance being satisfied.

\section{Data Availability}

The data used to support the findings of this study are included within the article.

\section{Conflicts of Interest}

The authors declare that they have no conflicts of interest.

\section{Acknowledgments}

This work was supported by the National Natural Science Foundation of China (grant no. 51805430) and the Natural Science Basic Research Program of Shaanxi (grant no. 2019JQ-365).

\section{References}

[1] Y. Rahmat-Samii and A. C. Densmore, "Technology trends and challenges of antennas for satellite communication systems," IEEE Transactions on Antennas and Propagation, vol. 63, no. 4, pp. 1191-1204, 2015.

[2] P. Lian, C. Wang, B. Xiang, Y. Shi, and S. Xue, "Gradientbased optimization method for producing a contoured beam with single-fed reflector antenna," Journal of Systems Engineering and Electronics, vol. 30, no. 1, pp. 22-29, 2019.

[3] M. Mahajan, R. Jyoti, K. Sood, and S. B. Sharma, "A method of generating simultaneous contoured and pencil beams from single shaped reflector antenna," IEEE Transactions on Antennas and Propagation, vol. 61, no. 10, pp. 5297-5301, 2013.

[4] R. C. Gupta, S. K. Sagi, K. P. Raja, N. K. Sharma, and R. Jyoti, "Shaped prime-focus reflector antenna for satellite communication," IEEE Antennas and Wireless Propagation Letters, vol. 16, pp. 1945-1948, 2017.

[5] M. W. Thomson, "AstroMeshTM deployable reflectors for $\mathrm{Ku}$ - and Ka- band commercial satellites," in Proceedings of the Twentyth AIAA International Communication Satellite Syst. Conf., Montreal, QC, USA, May 2002.

[6] G. Yang, D. Yang, Y. Zhang, and J. Du, "Form-finding design of cable-mesh reflector antennas with minimal length configuration," Aerospace Science and Technology, vol. 63, pp. 9-17, 2017.

[7] J. R. de Lasson, M. Zhou, and C. Cappellin, "Large deployable antennas benchmark for contoured beam mission in C band," in Proceedings of the 12th European Conference on Antennas and Propagation, pp. 1-5, London, UK, April 2018.

[8] H. Tanaka, "Design optimization studies for large-scale contoured beam deployable satellite antennas," Acta Astronautica, vol. 58, no. 9, pp. 443-451, 2006.

[9] S. Zhang, B. Duan, H. Bao, and P. Lian, "Sensitivity analysis of reflector antennas and its application on shaped geo-truss unfurlable antennas," IEEE Transactions on Antennas and Propagation, vol. 61, no. 11, pp. 5402-5407, 2013.

[10] S. Zhang, B. Y. Duan, and D. Yang, "Sensitivity analysis of faceted reflector antennas and a contour beam synthesis technology," IET Microwaves, Antennas \& Propagation, vol. 8, no. 14, pp. 1228-1235, 2014.

[11] G. Yang, Y. Zhang, A. Tang, and Y. Li, "A design approach for AstroMesh-type contoured-beam reflector antennas," IEEE Antennas and Wireless Propagation Letters, vol. 17, no. 6, pp. 951-955, 2018.

[12] Y. Zhang, B. Dong, G. Yang, D. Yang, and S. Zhang, "Design technique for a shaped-reflector antenna with a three-layer cable net structure," IEEE Transactions on Antennas and Propagation, vol. 69, no. 1, pp. 109-121, 2021.

[13] Y. Rahmat-Samii, "A comparison between GO/aperture-field and physical-optics methods of offset reflectors," IEEE Transactions on Antennas and Propagation, vol. 32, no. 3, pp. 301-306, 1984.

[14] W. Xu, B. Y. Duan, P. Li, N. Hu, and Y. Qiu, "Multiobjective particle swarm optimization of boresight error and transmission loss for airborne radomes," IEEE Transactions on Antennas and Propagation, vol. 62, no. 11, pp. 5880-5885, 2014.

[15] S. Morterolle, B. Maurin, J. Quirant, and C. Dupuy, "Numerical form-finding of geotensoid tension truss for mesh reflector," Acta Astronautica, vol. 76, pp. 154-163, 2012. 